\title{
Weak measurement of cotunneling time
}

\author{
Alessandro Romito ${ }^{1}$ and Yuval Gefen ${ }^{2}$ \\ ${ }^{1}$ Dahlem Center for Complex Quantum Systems and Fachbereich Physik, Freie Universität Berlin, 14195 Berlin, Germany \\ ${ }^{2}$ Department of Condensed Matter Physics, Weizmann Institute of Science, Rehovot, Israel
}

(Received 16 December 2013; revised manuscript received 14 July 2014; published 14 August 2014)

\begin{abstract}
Quantum mechanics allows the existence of "virtual states" that have no classical analog. Such virtual states defy direct observation through strong measurement, which would destroy the volatile virtual state. Here, we show how a virtual state of an interacting many-body system can be detected employing a weak measurement protocol with post-selection. We employ this protocol for the measurement of the time it takes an electron to tunnel through a virtual state of a quantum dot (cotunneling). Contrary to classical intuition, this cotunneling time is independent of the strength of the dot-lead coupling and may deviate from that predicted by time-energy uncertainty relation. Our approach, amenable to experimental verification, may elucidate an important facet of quantum mechanics which hitherto was not accessible by direct measurements.
\end{abstract}

DOI: 10.1103/PhysRevB.90.085417

PACS number(s): 73.63.Kv, 03.65.Ta, 73.23.Hk

\section{INTRODUCTION}

An important aspect of quantum mechanics is the existence of states that have no classical analog. Such "virtual states" cannot exist in classical physics as they violate energy conservation. It is commonly suggested that their presence within quantum mechanics as short-lived states is allowed by the uncertainty principle [1] $\Delta t \Delta E \sim \hbar$. These states, being volatile, are destroyed by a strong measurement, and are therefore inaccessible to direct detection. By contrast, weak measurement, along with its weak backaction, may provide us with a nondestructive probe into virtual states. In fact, weak measurement based protocols with post-selection [weak values (WV)] [2] have been employed with remarkable success in explaining quantum paradoxes [3], detecting and amplifying weak signals [4,5], directly measuring a wave function [6], and devising protocols for quantum states discrimination [7].

The primary goal of this paper is to extend the utility of WVs to the arena of many-body states, specifically probing virtual many-body states. This task is accomplished here for the first time by specifically considering the process of cotunneling $[8,9]$. In the latter, electrons are transported between a source $(S)$ and a drain $(D)$ through a quantum dot (QD); the QD is tuned such that the addition of an extra charge to it is classically forbidden (the Coulomb blockade regime) [10]. Nevertheless, an electron can enter the QD and later exit, forming a (short-lived) virtual many-body correlated state. This cotunneling process is qualitatively different from a single-particle tunneling under the barrier. We design a weak value protocol, amenable to experimental test, tailored to measure the lifetime of such a many-body virtual state. We anticipate that our demonstration of feasibility of such a protocol will pave the road to the study of a host of many-body problems that involve virtual states.

The second goal of our work is the study of the specifics of cotunneling lifetime. The lifetime of a virtual state associated with the tunneling of a single particle under a barrier has been studied extensively with a variety of approaches [12-20]. There are several time scales involved in this process: the dwell time $\tau_{\text {dwell }}$ marks the lifetime of the virtual state regardless of whether the electron is eventually transmitted (to $D$ ) or reflected (to $S$ ); the traversal time is the lapse between the disappearance of an electron from $S$ and its appearance in
$D$. For the tunneling of a single particle it has been shown [14] that the time is related to the imaginary velocity of the particle under the barrier. Determining the traversal time in the many-body cotunneling case poses a more difficult challenge, elucidated in the following.

Here, we show that naive expectations based on analogy with a single-particle tunneling are unfounded. Strikingly, we also find that the cotunneling time $\tau_{\text {cot }}$ may not be related to the time-energy uncertainty relation [11] $\Delta E \tau_{\text {cot }} \sim \hbar$, where $\Delta E$ represents the violation of energy conservation in the virtual state. Finally, we note that, while by classical intuition the transmission time through a QD should depend on the dot-lead tunneling matrix element, this turns out not to be the case here. Our results are summarized in Table I. We find that $\tau_{\text {cot }}$ depends parametrically on whether the cotunneling is dominated by elastic or inelastic processes.

In the following, after defining our model of a detector weakly coupled to a quantum dot tuned to the cotunneling regime, we review the measurement time in the regime of sequential tunneling, where the transport occurs through classical probability rates of tunneling in and out of the dot. Employing the same setup, we present a semiheuristic procedure through which we define the cotunneling time. We then show that this definition coincides with the quantity obtained relying on a weak-value-based protocol. We finally compute the cotunneling time in the various relevant parameter regimes.

\section{MODEL AND SETUP}

Our setup [cf. Fig. 1(a)] consists of a system (a quantum dot weakly coupled to leads) and a detector [a quantum point contact (QPC)]. The detector measures the system through the electrodynamic coupling between them. The way this setup is defined it is suitable to discuss both transport that involves real processes (sequential tunneling) as well as virtual processes (cotunneling). The relevance of many-body states is self-evident here. The corresponding Hamiltonian is given by

$$
H=H_{0}+H_{T}+H_{\text {int }}+\mathcal{H}_{\text {detector }},
$$

where $H_{0}$ represents the isolated (but voltage-biased) QD and the uncoupled source $(S)$ and drain $(D)$ leads; $H_{T}$ stands for 
TABLE I. Real and imaginary components of the cotunneling time $\tau_{\mathrm{WV}}$ for the inelastic and elastic cotunneling regimes. Listed are the respective average cotunneling currents too. The results are written in terms of the dot-leads conductance $G_{\alpha}=e^{2} v_{\alpha} v_{0}\left|\gamma^{\alpha}\right|^{2} /(2 \pi \hbar)$, with $v_{\alpha(0)}$ being the density of states in the lead (dot); the relevant energy scales in the dot are the level spacing $\delta$, the Thouless energy $E_{\mathrm{Th}}$, the charging energy $E_{C}$, and the applied voltage bias $e V .\left|\mathbf{x}_{S}-\mathbf{x}_{D}\right|$ is the distance between the source and drain contacts, $L$ the linear size of the quantum dot. The results in the last two rows are explicitly for the two-dimensional (2D) case.

\begin{tabular}{lccc}
\hline \hline & $\operatorname{Re}\left\{\tau_{\mathrm{WV}}\right\}=\tau_{\mathrm{dwell}}$ & $\operatorname{Im}\left\{\tau_{\mathrm{WV}}\right\}= \pm \tau_{\mathrm{cot}}$ & $\langle I\rangle /\left(\frac{G^{(S)} G^{(D)}}{12 \pi e^{2}} V\right)$ \\
\hline Inelastic & 0 & $\frac{\hbar}{E_{C}}$ & $\frac{(e V)^{2}}{E_{C}^{2}}$ \\
Elastic & 0 & $-\frac{1}{2} \frac{\hbar}{E_{C}}$ & $\frac{3 \delta}{E_{c}}$ \\
$E_{C} \ll E_{\mathrm{Th}}$ & 0 & $-\frac{3 \hbar}{2 E_{C}} \ln ^{-1}\left(\frac{L^{2} E_{\mathrm{Th}}}{\left|\mathbf{x}_{S}-\mathbf{x}_{D}\right|^{2} E_{C}}\right)$ & $\frac{\delta}{E_{\mathrm{Th}}} \ln ^{3}\left(\frac{L^{2} E_{\mathrm{Th}}}{\left|\mathbf{x}_{S}-\mathbf{x}_{D}\right|^{2} E_{C}}\right)$ \\
$E_{\mathrm{Th}} \ll E_{C} \ll \frac{L^{2} E_{\mathrm{Th}}}{\left|\mathbf{x}_{S}-\mathbf{x}_{D}\right|^{2}}, 2 \mathrm{D}$ & 0 & $-\frac{\hbar}{E_{C}}$ & $\frac{3 \delta E_{\mathrm{Th}}}{4 E_{C}^{2}}$ \\
$E_{\mathrm{Th}} \lesssim \frac{L^{2} E_{\mathrm{Th}}}{\left|\mathbf{x}_{S}-\mathbf{x}_{D}\right|^{2}} \ll E_{C}, 2 \mathrm{D}$ & 0 & \\
\hline \hline
\end{tabular}

the dot-leads tunnel coupling and $H_{\text {detector }}+H_{\text {int }}$ describe the detector dynamics and its interaction with the QD. Specifically, the part of the Hamiltonian concerning the system (the QD and the leads) consists of the following terms:

$$
H_{0}=\sum_{\alpha=S, D} \sum_{k} \epsilon_{\alpha, k} c_{\alpha, k}^{\dagger} c_{\alpha, k}+\sum_{h} \epsilon_{0, h} d_{h}^{\dagger} d_{h}+U .
$$

It describes the isolated QD, and the uncoupled source and drain leads. The single-particle terms for the source $(S)$ and drain $(D)$ leads and the QD are expressed in terms of the fermionic field operators $c_{S, k}, c_{D, k}, d_{h}$. The charging energy contribution [10]

$$
U=E_{C}\left(N_{S}+N_{D}-N_{g}\right)^{2}-\frac{e V}{C_{\Sigma}}\left(C_{S} N_{S}-C_{D} N_{D}\right),
$$

with $N=\sum_{\alpha} d_{\alpha}^{\dagger} d_{\alpha}$, characterized by the energy $E_{C}$, depends on the charge entering the dot from the left (right) lead $N_{S(D)}$. $N=N_{S}+N_{D}$ is the extra charge on the dot. Equation (3) provides the explicit dependence of the charging energy $U$ on dot-source $C_{S}$, dot-drain $C_{D}$, and dot-gate $C_{g}$ capacitances
( $C_{\Sigma}=C_{S}+C_{D}+C_{g}$ is the total capacitance), as well as on the source-drain voltage bias $e V$, and on the gate voltage, through $N_{g}=V_{g} /\left(e C_{g}\right)$.

The leads QD tunneling operator $H_{T}=\sum_{\alpha=S, D} T_{\alpha}+$ H.c., with $T_{\alpha}=\sum_{k, h} \gamma_{k, h}^{(\alpha)} d_{h}^{\dagger} c_{\alpha, k}$, is written in terms of source and drain tunneling amplitudes $\gamma_{h, k}^{(\alpha)}$. The current operator in the QD is

$$
I=\partial_{t} \sum_{k} c_{D, k}^{\dagger} c_{D, k}=i\left(T_{D}-T_{D}^{\dagger}\right)
$$

Transport via virtual processes is $\propto \gamma^{4}$, and can be classified into inelastic and elastic cotunneling, corresponding to the state of the QD being modified or unmodified, respectively, following a tunneling event. In any case, the virtual occupation of the dot involves a many-electron correlated state.

The detection of the excess charge on the dot $e N$ is carried out by a quantum point contact capacitively coupled to the dot, which is routinely employed in experiments as a charge sensor [21-23]. The QPC is modeled as a scattering potential (a)

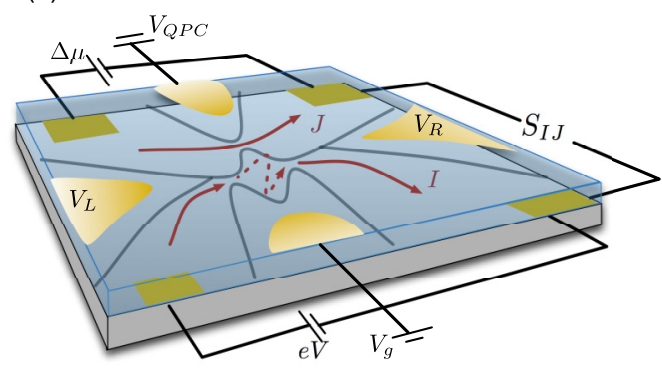

(b)

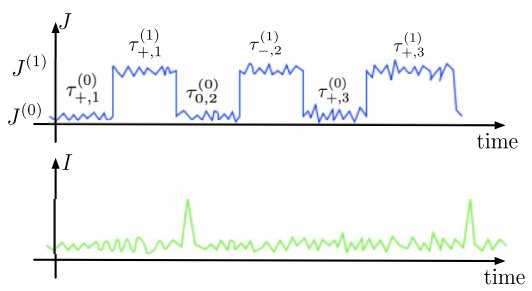

(c)

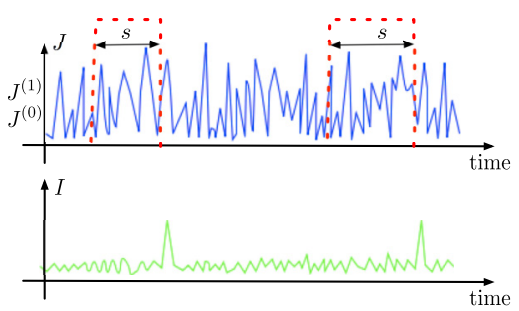

FIG. 1. (Color online) A measurement scheme of the cotunneling time. (a) Sketch of a quantum dot coupled to a QPC detector. The transmission through the QPC is affected by the presence of an extra electron in the QD. The top gates $V_{L}$ and $V_{R}$ control the tunneling rate to the dot, $V_{\mathrm{QPC}}$ the unperturbed transmission through the QPC, and $V_{g}$ the charging energy in the dot. $V_{g}$ allows us to tune the system from the sequential tunneling to the cotunneling regime. The transport through the QPC and the QD is controlled by the voltage bias $\Delta \mu$ and $e V$, respectively. The current-current correlation $S_{I J}$ is sensitive to the excess number of electrons $N$ in the dot. (b) Typical detector signal and current through the dot in the sequential tunneling regime, performing strong measurement with the QPC. The time an electron spends in the dot can be classified according to the occurrence of a subsequent positive current pulse in the drain $\left(\tau_{+, i}\right)$, or the absence thereof: back-reflection $\left(\tau_{-, i}\right)$. The average sequential tunneling time can be directly obtain by averaging over the durations $\left\{t_{+, i}^{(1)}\right\}$ of the relevant QPC signals. (c) Typical detector signal and current through the dot in the weak measurement regime. Straightforward classification of events as in (b) is not possible. The signal of the QPC preceding a pulse of current through the quantum dot has to be weighted by an appropriate weight function. The latter should account for the QPC current signal, which precedes and is directly related to the electron detected at the QD's drain terminal In the cotunneling regime, the interval between successive tunneling events is longer than the cotunneling time, hence, one may discard the weighting function. 
for impinging electrons through

$$
H_{\text {detector }} \equiv H_{\mathrm{QPC}}=\sum_{i=l, r} \sum_{k} v k a_{i, k}^{\dagger} a_{i, k},
$$

with $a_{i, k}$ being the annihilation operator for the left- $(i=l)$ and right- $(i=r)$ moving scattering states; here $v$ is the magnitude of the electron velocity. The addition of an extra electron to the $\mathrm{QD}(\langle N\rangle=1)$ results in the modification of the QPC scattering potential. The coupling to the QD is specified by $H_{\text {int }}=N X$ $[24,25]$ with

$$
X=\frac{v(\delta \kappa+i u)}{L \sqrt{\kappa(1-\kappa)}} \sum_{k, h} a_{r, k}^{\dagger} a_{l, h}+\text { H.c. }
$$

It describes a general complex backscattering amplitude due to the modification of the scattering potential due to the occupation of the QD by an extra electron. The QPC has a chemical potential bias $\Delta_{\mu}$, which defines the detector's bandwidth and is assumed to be the largest energy scale in the problem. The operator associated with the QPC signal is then the current through the QPC [24,26]

$$
\begin{aligned}
J=\frac{e v}{L} \sum_{k, p} & {\left[\sum_{i=l, r} \kappa(-1)^{i} a_{i, k}^{\dagger} a_{i, p}\right.} \\
& \left.+\sqrt{\kappa(1-\kappa)}\left(i e^{-i(k-p) x} a_{r, k}^{\dagger} a_{l, p}+\text { H.c. }\right)\right] .
\end{aligned}
$$

Here, $\kappa$ is the transmission probability through the QPC, $L$ is the QPC length, and $x$ plays the role of a regularization parameter.

Various scales of charging energy. In view of the calculations of the cotunneling current and cotunneling time, we conveniently denote by $\left\langle U\left(T_{S}\right)\right\rangle$ the change of charging energy due to the tunneling of an extra charge from the source into the dot, and by $\left\langle U\left(T_{S}^{\dagger}\right)\right\rangle$ the corresponding change due to the exit of a charge to the source. With the equivalent notation of the tunneling to/from the drain $(D)$, we can generally denote the modification of the charging energy due to a certain sequence of tunneling events as $\left\langle U\left(T_{\alpha}, \ldots, T_{\beta}\right)\right\rangle$ with $\alpha, \beta=S, D$. In the Coulomb blockade regime where the charging energy $E_{c}$ is the largest energy scale of the quantum dot's dynamics, we consider only the states with $N=0,1$ excess electrons in the dot. Then, we obtain the relevant charging energies in the cotunneling processes directly from Eq. (3):

$$
\begin{aligned}
\left\langle U\left(T_{S}\right)\right\rangle & \equiv E_{+l}, \quad\left\langle U\left(T_{D}^{\dagger}\right)\right\rangle \equiv E_{-r}, \\
\left\langle U\left(T_{D}\right)\right\rangle & =E_{+l}+e V, \quad\left\langle U\left(T_{S}^{\dagger}\right)\right\rangle=E_{-r}+e V, \\
\left\langle U\left(T_{S} T_{D}^{\dagger}\right)\right\rangle & =-e V, \quad\left\langle U\left(T_{S}^{\dagger} T_{D}\right)\right\rangle=e V, \\
\left\langle U\left(T_{S} T_{S} T_{D}^{\dagger}\right)\right\rangle & =-e V+E_{+l}, \\
\left\langle U\left(T_{D} T_{D} T_{s}^{\dagger}\right)\right\rangle & =2 e V+E_{+l}, \\
\left\langle U\left(T_{S}^{\dagger} T_{S}^{\dagger} T_{D}\right)\right\rangle & =2 e V+E_{-r}, \\
\left\langle U\left(T_{D}^{\dagger} T_{D}^{\dagger} T_{S}\right)\right\rangle & =-e V+E_{-r} .
\end{aligned}
$$

Following, we will focus on the limit $e V \ll E_{-r} \ll E_{+l}$, in which cotunneling is dominated by particlelike processes rather than holelike processes; hereafter, we simply set $E_{+l} \equiv E_{C}$.

\section{A HEURISTIC APPROACH}

Before addressing the cotunneling regime, let us discuss how the detection scheme of Fig. 1 works in the sequential tunneling regime, which is a real (nonvirtual) process. Referring to Fig. 1(b), we note that the entry of the $i$ th electron into the QD may result in a successful (unsuccessful) sojourn $\tau_{+, i}^{(1)}$ $\left(\tau_{-, i}^{(1)}\right)$, at the end of which the electron is transmitted to the drain (is backscattered). The current through the QPC, $J$, is a two-valued signal, where the two values $J^{(0)}$ and $J^{(1)}$ are associated with the absence or presence of an extra electron on the QD. Noting that a peak in the current through the QD, $I$, signals a successful tunneling event (we neglect processes where the electrons hop from the drain to the dot), one can easily extract the time of sequential tunneling $\left\langle\tau^{(1)+}\right\rangle_{\text {seq }}$ from

$$
\left\langle J_{+}\right\rangle=\left(J^{(1)}-J^{(0)}\right)\left\langle\tau_{+}^{(1)}\right\rangle_{\mathrm{seq}}\langle I\rangle / e,
$$

which defines the tunneling time in terms of the excess current in the QPC conditional to the occurrence of a tunneling event through the QD $\left\langle J_{+}\right\rangle$and the average tunneling current $\langle I\rangle$. A rate equation analysis reveals that $\left\langle\tau_{+}^{(1)}\right\rangle_{\text {seq }}$ depends on the source and drain tunneling rates $\Gamma_{S}$ and $\Gamma_{D}$, respectively, yielding $\left\langle\tau_{+}^{(1)}\right\rangle_{\text {seq }}=1 /\left(\Gamma_{S}+\Gamma_{D}\right)$.

While Eq. (9) is straightforwardly applicable to experiments with strong QD-detector coupling [23], $\left\langle J_{+}\right\rangle$cannot be directly addressed in the weak measurement regime. The signal $J^{(1)}-J^{(0)}$ is then masked by quantum noise [cf. Fig. 1(c)]; it is not possible to uniquely determine the duration of each interval $\tau_{+. i}^{(1)}$. This hurdle can be overcome by introducing a (Poissonian) probability distribution $p(t)$ for the time intervals $\left\{\tau_{+, i}^{(1)}\right\}$. Evidently, $p(t)$ depends on $\left\langle\tau_{+}^{(1)}\right\rangle_{\text {seq. The sequential }}$ tunneling time may be obtained through an average over such a distribution, as

$\left\langle\tau_{+}^{(1)}\right\rangle_{\text {seq }}=\lim _{T \rightarrow \infty} \frac{\int_{0}^{T} d t \int_{0}^{t} d s P(s)\left[J(t-s)-J^{(0)}\right] I(t)}{T\langle I\rangle\left(J^{(1)}-J^{(0)}\right)}$,

where $P(t)=1-\int_{0}^{t} d s p(s)$ is the probability the electron entering the dot at $t=0$ remains in the dot at time $t$. This is a self-consistent equation for $\left\langle\tau_{+}^{(1)}\right\rangle_{\text {seq. }}$. A direct calculation shows that Eq. (10) leads to the same results as Eq. (9) (cf. Appendix A).

We now consider the case of cotunneling. Here, we generalize Eq. (10) employing quantum mechanical currentcurrent correlations. We stipulate that these correlations decay in time faster than the time interval between two consecutive cotunneling events, hence, we may neglect the cutoff due to the $p(s)$, and replace $J^{(0)}$ by the average $\langle J\rangle$. This relates the cotunneling time to the current-current correlation function $S_{I J} \equiv \int_{0}^{\infty} d s\langle I(t)[J(t-s)-\langle J\rangle]\rangle$ through

$$
\left\langle\tau_{+}^{(1)}\right\rangle_{\cot }=\frac{S_{I J}}{\langle I\rangle\left(J^{(1)}-\langle J\rangle\right)} .
$$

It is worth noting that the integration in Eq. (11) is only over positive times.

Evidently, to evaluate the time obtained in the cotunneling regime, a microscopic treatment of the problem is due. For 
this we employ the Hamiltonian in Eq. (1)), and evaluate the averages in the Keldysh formalism [27,28]. To this goal a time ordering of operators $\mathcal{T}_{K}$ is introduced on a time contour consisting of two branches corresponding to forwardor backward-in-time parts of the contour. The operators are labeled by a subscript,+- depending on whether they belong to the former or the latter branch of the contour. To first order in perturbation in $H_{\text {int }}$, the correlator $S_{J I}$ reads as

$$
\begin{aligned}
S_{J I}= & -i \int_{0}^{\infty} d \tau \int_{-\infty}^{\infty} d s\left[\left\langle\mathcal{T}_{K}\left[I_{-}(t+\tau) N_{+}(s)\right]\right\rangle g_{++}(s-t)\right. \\
& \left.-\left\langle\mathcal{T}_{K}\left[I_{-}(t+\tau) N_{-}(s)\right]\right\rangle g_{-+}(s-t)\right],
\end{aligned}
$$

where $\quad g_{++}\left(t-t^{\prime}\right) \equiv\left\langle\mathcal{T}_{K}\left[J_{+}\left(t^{\prime}\right) X_{+}(t)\right]\right\rangle, \quad g_{-+}\left(t-t^{\prime}\right) \equiv$ $\left\langle\mathcal{T}_{K}\left[J_{-}\left(t^{\prime}\right) X_{+}(t)\right]\right\rangle$. In the limit considered here, where $\Delta \mu$ is the largest energy scale in the problem, one obtains $g_{-+}(t-$ $\left.t^{\prime}\right)=g_{++}\left(t-t^{\prime}\right)=(e \Delta \mu) /(2 \pi)(i \delta \kappa+u) \delta\left(t-t^{\prime}+\eta\right)$, and finally

$$
\left\langle\tau_{+}^{(1)}\right\rangle_{\cot }=\operatorname{Re}\left\{\tau_{\mathrm{WV}}\right\}-(u / \delta \kappa) \operatorname{Im}\left\{\tau_{\mathrm{WV}}\right\},
$$

where $\tau_{\mathrm{WV}}$ is an intrinsic quantity of the system with the dimensions of time

$$
\tau_{\mathrm{WV}}=\frac{\int_{0}^{\infty}\langle I(t)[N(t-s)-\langle N\rangle]\rangle}{\langle I\rangle} .
$$

In fact, $\tau_{\mathrm{WV}}$ is the complex time obtained by a direct application of a weak value protocol to the cotunneling time.

\section{COTUNNELING TIME FROM THE WEAK VALUE FORMALISM}

In this section, we present the result for the cotunneling time as obtained through a direct application of the weak value formalism. In complete analogy with the problem of singleparticle tunneling time [19], $\tau_{\mathrm{WV}}$ is obtained with the aid of an ideal detector whose dynamics is trivial $\left(H_{\text {detector }}=0\right)$.

The result is obtained employing a simple model in which the detector is modeled as a pointer coupled via $H=\lambda \hat{p} N$, $N$ being the excess charge in quantum dot (measured in units of the electron charge $e ; N$ may assume the values +1 or 0 ), and $q$ the position of the detector pointer (initially at $\langle q\rangle=0)$ with $[q, p]=i \hbar$. The detector is assumed to have no internal dynamics $\left(H_{\operatorname{det}}=0\right)$. Measuring $\hat{q}$ at a time $\Delta t$ leads to $\langle q\rangle=\lambda \int^{\Delta t} d s N(s)$. One can interpret this expression to obtain either (i) the time-averaged charge in the dot $e\langle N\rangle=e\langle q(t)\rangle / \lambda \Delta t$, where $\Delta t$ is the duration of the measurement, or (ii) the average time the particle spends in the dot, $\tau=\langle q\rangle / \lambda$. In the latter interpretation, it is important that the charge exists in quantized units of $e$, and that during the measurement time $\Delta t$ at most a single cotunneling event takes place. In the case of sequential tunneling, this procedure results in $\langle q\rangle / \lambda$ being exactly the $d$ well time (as distinct from the cotunneling time) of the particle in the QD. We assume this is a valid measurement of the dwell time also in the regime of cotunneling.

In order to address the time the particle spends in the dot conditional to a later successful cotunneling event (which takes the particle to the drain), we can make use of the weak value formalism [2]. The signal in the detector, conditional to a successful cotunneling through the QD, is expressed as $\tau_{\text {cot }}={ }_{f}\langle q(t)\rangle_{0} / \lambda=\operatorname{Re}\left\{{ }_{f}\left\langle\tau_{\mathrm{WV}}\right\rangle_{0}\right\}$, where $f$ indicates that the average has to be taken conditional to the post-selection of a certain state $|f\rangle$ of the system. In the weak measurement regime, $\tau_{\mathrm{WV}}$ is the weak value of the measured observable, hence,

$$
\tau_{\mathrm{WV}}=\int^{t}{ }_{f}\langle N(s)\rangle_{0}=\frac{\int^{t} d s\left\langle\Pi_{f}(t) N(s)\right\rangle}{\left\langle\Pi_{f}(t)\right\rangle},
$$

where $\Pi_{f}$ is the projection into the post-selected state.

In order to specify the post-selection of the cotunneling process, we consider a simple picture where an electron, initially in the source reservoir, can eventually reach the drain. The correlations between subsequently impinging electrons are neglected, as well as the virtual occupation of the dot by processes originating from the drain.

The projector onto the post-selected state (i.e., successful cotunneling) at a time $\Delta t$ is $N_{D}(\Delta t)$ (where the excess particle number $N_{D}$ is measured from the reference value before the cotunneling process started taking place). The post-selection is, in fact, the result of a continuous measurement over the interval $\Delta t$, which accounts for all possible arrival times of the electron in the drain during the time interval $[0, \Delta t]$. This can be properly taken into account by summing the probability of tunneling at any time and noting that it can be expressed via the current operator at the drain as $N_{D}(\Delta t)=\int^{\Delta t} d t I(t)$. We therefore implement the post-selection operator as $\Pi_{f}=$ $\int^{\Delta t} d t I(t)$. In doing so, we note that the detection of an electron in the drain at time $t<\Delta t$ consists of a strong (post-selection) measurement. Therefore, in assessing the weak value, we need to account only for weak measurements that preceded that strong measurement at time $t$. This is implemented by constraining the correlation between $I(t)$ and $N(s)$ for time intervals such that $s<t$. Finally, since we are dealing with a steady state, the correlations $\langle N(s) I(t)\rangle$ depend only on the time difference $t-s$ and we can write

$$
\tau_{\mathrm{WV}}=\frac{\int_{0}^{\infty} d s\langle I(t) N(t-s)\rangle}{\langle I\rangle} .
$$

The resulting complex $\tau_{\mathrm{WV}}$ encodes the information on the physical times involved in the cotunneling process. Notably, the measured QPC-QD current correlation provides access only to a combination of the real and imaginary parts of $\tau_{\mathrm{WV}}$ (which depends on nonuniversal details of the variation of the QPCs transparency as function of the QDs occupation). The individual parts can be obtained through detailed tomography of $\tau_{\mathrm{WV}}$. To shed light on the physical meaning of $\tau_{\mathrm{WV}}$, it is instructive to first discuss the analogous complex tunneling time in the context of a single-particle tunneling.

Analysis of a complex $\tau_{\mathrm{WV}}$ for a single-particle tunneling. Here, we review the analysis of the tunneling time of a single particle (single-particle opaque barrier), through a weak value protocol. This serves as a benchmark for the analysis of the equivalent cotunneling time. The issue of the time of single-particle tunneling has been discussed extensively in the literature in a variety of approaches: both vis-a-vis single-particle tunneling in real space $[12-15,18]$, and to Landau-Zener tunneling in energy space [16]. A weak measurement approach to this problem [19] gives rise to a tunneling time and a reflection time $\tilde{\tau}_{\text {tun }}$ and $\tilde{\tau}_{\text {ref }}$, respectively, 
both being complex. The weighted dwell time under the barrier is then $\tilde{\tau}_{\text {dwell }}=T \tilde{\tau}_{\text {tun }}+(1-T) \tilde{\tau}_{\text {ref }}(T$ being the transmission probability). Quite remarkably, this last equality holds for the complex tunneling and reflection times. The physical times of this problem are the dwell time $\tilde{\tau}_{\text {dwell }}=\operatorname{Re}\left\{\tilde{\tau}_{\text {tun }}\right\}$ and the traversal time under the barrier $\tilde{\tau}_{T}=\left[\tilde{\tau}_{\text {dwell }}^{2}+\left(\operatorname{Im}\left\{\tilde{\tau}_{\text {tun }}\right\}\right)^{2}\right]^{1 / 2}$ $[15,19]$. In the limit of a high thin barrier $\tilde{\tau}_{\text {dwell }}$ vanishes (most reflected particles spend a negligible time under the barrier), hence, $\tilde{\tau}_{T}=\left|\operatorname{Im}\left\{\tilde{\tau}_{\text {tun }}\right\}\right|$. This agrees with the result of Ref. [15].

\section{MICROSCOPIC CALCULATION}

We now turn to calculate the cotunneling time in Eq. (14).

While the result for the cotunneling current is known $[8,9]$, it is the charge-current correlation function of the system that encodes information on the cotunneling time. For simplicity, we tune the gate voltage such that transport through the quantum dot is dominated by particlelike cotunneling. Our analysis addresses the limit where the temperature is smaller than the source-drain voltage. Different contributions to the current are described by Feynman diagrams and are conveniently grouped into elastic and inelastic contributions [cf. Fig. 2(a)] [8]. Correspondingly, we group the contributions to the correlator $\langle I(t)[N(t-s)-\langle N\rangle]\rangle$ into two sets. The results for the complex cotunneling time will differ depending on whether the cotunneling is dominated by elastic or inelastic processes. Examples of diagrams that contribute to $\langle I\rangle$ and $\langle I(t)[N(t-s)-\langle N\rangle]\rangle$ are depicted in Fig. 2. All in all, we have 64 different diagrams; when dealing with particledominated cotunneling and considering the zero-temperature limit, the number of diagrams is reduced to eight (four elastic processes and four inelastic processes), as shown in
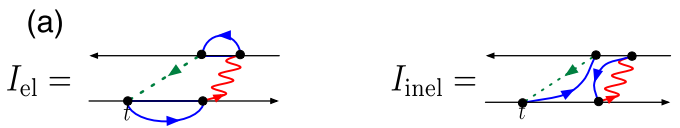

(b)
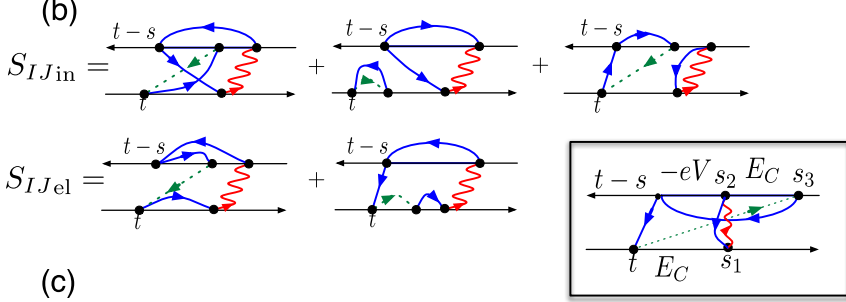

(c)

$\hat{\jmath}=\sum_{h} d_{h}^{\dagger} d_{h} \quad \hat{\xi}=\sum_{k, h} \gamma_{k, h}^{(S)} \quad \hat{\jmath}=\sum_{k, h} \gamma_{k . h}^{(D)}$

$\longrightarrow{ }^{0}=\left\langle d_{h}(t) d_{h}^{\dagger}(0)\right\rangle \quad{ }^{0} \mathbf{r n}^{t}=\left\langle c_{S, k}(t) c_{S, k}^{\dagger}(0)\right\rangle \quad{ }^{0} \rightarrow .^{t}=\left\langle c_{D, k}(t) c_{D, k}^{\dagger}(0)\right\rangle$

FIG. 2. (Color online) Feynman diagrams for $\langle I\rangle$ and $S_{I J}$. Elastic and inelastic contributions to the cotunneling current $\langle I\rangle$ [panel (a)], and to the current-current correlator $S_{I J}$ [panel (b)], in the zerotemperature particle-dominated regime. The propagators and vertices constituting the diagrams are defined in panel (c). Inset: An example of a diagram neglected in the zero-temperature particle-dominated limit. Here, we indicate explicitly the time and charging energy labelings along the time contour, as dictated by the Feynman rules (cf. Appendix B3).
Appendix B. The nonvanishing diagrams in this limit are depicted in Fig. 2(b), and are evaluated in Appendix B2. The expressions for $\langle I(t)[N(t-s)-\langle N\rangle]\rangle$ and $\langle I\rangle$ in Eqs. (B8), (B7), (B23), and (B24) are finally substituted into Eq. (14) to find

$$
\tau_{\mathrm{WV}}=\mp \frac{i}{2} \partial_{E_{C}} \ln I
$$

with + and - for inelastic and elastic cotunneling, respectively. An equivalent expression yielding the traversal time in terms of the logarithmic derivative of the transmission probability holds for the noninteracting case, where, for tunneling through a square potential barrier, it reads as $\tau_{\mathrm{WV}}=-\partial_{V_{0}}[\arg (t)]+$ $\frac{i}{2} \partial_{V_{0}} \ln \left(t^{*} t\right)$ [17]. Here, $V_{0}$ is the barrier height and $t$ the transmission amplitude. Our result extends the validity of such an equation to interacting systems.

Equation (17) is rather general, and does not depend on the specifics of the electronic dynamics in the quantum dot. However, in order to obtain specific expressions for $\tau_{\mathrm{WV}}$, we specify the dot to be in the diffusive limit $L>\left|\mathbf{x}_{\mathbf{S}}-\mathbf{x}_{\mathbf{D}}\right| \gg l$, where $L$ is the linear size of the quantum dot, $l$ the elastic mean-free path, and $\mathbf{x}_{S}, \mathbf{x}_{D}$ the position of the source and drain contacts, respectively. As shown in Appendix B, the cotunneling current can then be expressed in terms of the diffuson propagator [29] between the source and the drain points $\mathcal{D}_{\omega}\left(\mathbf{x}_{S}, \mathbf{x}_{D}\right) \equiv$ $v_{0} \mathcal{S}^{2}\left\langle\psi_{\alpha}\left(\mathbf{x}_{S}\right) \psi_{\alpha}^{*}\left(\mathbf{x}_{D}\right) \psi_{\beta}\left(\mathbf{x}_{D}\right) \psi_{\beta}^{*}\left(\mathbf{x}_{S}\right)\right\rangle$, where $\psi_{\alpha}(\mathbf{x})$ is the wave function of the $\alpha$-energy level of the dot with energy $\epsilon_{\alpha}$, $\omega=\epsilon_{\alpha}-\epsilon_{\beta}, v_{0}$ and $\mathcal{S}$ are respectively the density of states and the area of the dot, and the average is intended over the different statistical realizations of disorder.

In this case, the cotunneling current reads as [8,9,25,29]

$$
\begin{aligned}
I_{\mathrm{in}}= & \frac{G^{(S)} G^{(D)}}{12 \pi e^{2}} \frac{(e V)^{2}}{E_{C}^{2}} V \\
I_{\mathrm{el}}= & \frac{G^{(S)} G^{(D)}}{4 \pi^{2} \nu_{0} e^{2}} V \int_{0}^{\infty} d \omega \frac{\mathcal{D}_{\omega}\left(\mathbf{x}_{S}, \mathbf{x}_{D}\right)+\mathcal{D}_{-\omega}\left(\mathbf{x}_{S}, \mathbf{x}_{D}\right)}{\omega} \\
& \times \ln \left(1+\frac{\omega}{E_{C}}\right)
\end{aligned}
$$

The elastic cotunneling current depends on the diffuson propagator, which is characterized by Thouless energy $E_{\mathrm{Th}} \sim D / \mathcal{S}$, proportional to the diffusion constant $D$. The cotunneling current depends on the ratio between $E_{\mathrm{Th}}$ and $E_{C}$. The cotunneling time in the various regimes is finally deduced directly from Eq. (17) and the corresponding expression for the cotunneling current [Eqs. (B9), (B11), (B13), and (B16)]. The results are listed in Table I.

We note here that, while the cotunneling current generally depends parametrically on the various energy scales in the different regimes, the cotunneling time is $\tau_{\text {cot }} \sim \hbar /\left(E_{C}\right)$ in all cases except for elastic cotunneling with very close contacts $E_{\mathrm{Th}} \ll E_{C} \ll \frac{L^{2} E_{\mathrm{Th}}}{\left|\mathbf{x}_{S}-\mathbf{x}_{D}\right|^{2}}$, where it is given by

$$
\tau_{\mathrm{WV}}=-i \frac{3}{2 E_{C}} \ln ^{-1}\left(\frac{D}{\pi^{2}\left|\mathbf{x}_{s}-\mathbf{x}_{D}\right|^{2} E_{C}}\right) .
$$

This shows explicitly that the cotunneling time can be parametrically different from the estimation obtained via the uncertainty principle. In particular, the particle can spend a time shorter than $1 / E_{C}$ in the dot. This is in striking contrast 
with the results for tunneling of a single particle through a barrier.

\section{DISCUSSION AND SUMMARY}

For a single-particle tunneling through an opaque barrier, it has been shown $[15,19]$ that the traversal time is given by the imaginary part of the complex weak value time; the real part turned out to be the dwell time, and was found to be vanishing. In the present analysis, we consistently find a vanishing dwell time $\left(\operatorname{Re}\left\{\tau_{\mathrm{WV}}\right\}=0\right)$, while $\tau_{\text {cot }}=\left|\operatorname{Im}\left\{\tau_{\mathrm{WV}}\right\}\right|$ (cf. Table I). We also note that Eq. (17) has been established for single-particle tunneling (opaque barrier [17]). We have shown here that it remains valid for interacting systems. The emerging picture shows that the cotunneling traversal time is not simply given by the uncertainty time $\hbar / E_{C}$, but it can be logarithmically smaller in $E_{\mathrm{Th}} / E_{C}, E_{\mathrm{Th}}$ being the Thouless time of flight through the QD [29] (assuming, for example, diffusive dynamics in the dot). The dependence on the ballistic or diffusive real time of flight through the QD is very different for the single-particle tunneling: the time of the latter is determined by the imaginary velocity under the barrier [14].

We have presented our analysis on three levels. First, we have defined a realistic system-detector setup both in the sequential and cotunneling regimes, and related the correlation function of the system-detector currents to $\left\langle\tau_{+}^{(1)}\right\rangle_{\text {seq }}$ or the complex $\left\langle\tau_{+}^{(1)}\right\rangle_{\text {cot }}$ [Eqs. (9)-(11)]. These expressions are useful for processing experimental data, through (i) analysis of current-current correlations [Eqs. (10) and (11)] or (ii), for single-shot measurements, selective inclusion of signals of detector current conditional on the later detection of a current pulse through the QD [Eq. (9)]. Second, through a weak value analysis, we have addressed the meaning of the complex time $\tau_{\mathrm{WV}}$. This complete $\tau_{\mathrm{WV}}$ contains information about the dwell and the cotunneling times. Third, we have considered Eqs. (10) and (11) as a starting point for a first-principles calculation of $\tau_{\mathrm{WV}}$, pursued through a diagrammatic Keldysh perturbation theory. Our protocol is amenable of experimental verification. For a ballistic semiconducting QD whose linear size is $L=$ $0.15 \mu \mathrm{m}$, the electrons Fermi velocity $v_{F}=10^{6} \mathrm{~cm} / \mathrm{sec}$ and $E_{C}=20 \mu \mathrm{eV}, E_{\mathrm{Th}}$ and $E_{C}$ are comparable. One may design and tune the relevant gates to achieve the desired inequality between these two energies. Within a broader context, the analysis outlined here demonstrates the usefulness of such composite measurements protocols for a systematic, nondestructive study of many-body systems driven to a virtual state.

\section{ACKNOWLEDGMENTS}

We thank K. Ensslin, T. Ihn, and P. W. Brouwer for stimulating discussions. We acknowledge the support of GIF, BSF under Grant No. 2006371, and DFG under Grants No. RO 4710/1-1 and No. RO 2247/8-1.

\section{APPENDIX A: SEQUENTIAL TUNNELING TIME: A CLASSICAL CASE}

We discuss here the measurement of the sequential tunneling time through a weak measurement scheme. We show explicitly that the sequential tunneling time obtained through the weak measurement scheme as in Eq. (10) coincides with the result of a direct strong measurement Eq. (9). To this goal, we consider a simple model of transport through the QD, where tunneling of subsequent electrons are uncorrelated events. We assume a constant flux $f_{0}$ of electrons emitted from the source. For simplicity, we assume that one electron impinges on the dot in the time $T$, i.e., $f_{0}=1 / T$; hereafter $\Gamma_{S}, \Gamma_{D}$ are the dot-source and dot-drain tunnel rates, respectively.

To begin, we evaluate the probability of the events describing transport in the dot. Assuming that an electron enters the dot at $t=0$, and that the time for tunneling out is Poissonian distributed, the probability $P(t)$ that an electron remains in the dot until time $t$ is

$$
P(t)=e^{-\left(\Gamma_{S}+\Gamma_{D}\right) t} .
$$

It follows that the probability $p(t) d t$ that an electron remains in the dot until time $t$ and exits it in the time interval $[t, t+d t]$ is given by

$$
p(t) d t=e^{-\left(\Gamma_{S}+\Gamma_{D}\right) t}\left(\Gamma_{D}+\Gamma_{S}\right) d t .
$$

We notice also that $P(D \mid t)=\Gamma_{D} /\left(\Gamma_{S}+\Gamma_{D}\right)$, where $P(D \mid t)$ is the probability that, given that the particle exits the dot within the time interval $[t, t+d t]$, it does it through the drain. The analogous equation with $\Gamma_{D} \leftrightarrow \Gamma_{S}$ holds for the corresponding probability $p(S \mid t) d t$ in the case of electron exiting toward the source. The probability that the electron exits the dot in the time interval $[t, t+d t]$, given that this takes place through the drain's barrier, is determined through the Bayes theorem, leading to $p_{D}(t) d t \equiv p(t \mid D) d t=p(D \mid t) p(t) / P(D)$, where $P(D)=\Gamma_{D} /\left(\Gamma_{S}+\Gamma_{D}\right)$ is the total probability to exit to the drain, integrated over all times. This results in

$$
p_{D}(t) d t=p(t) d t={ }^{-\left(\Gamma_{S}+\Gamma_{D}\right) t}\left(\Gamma_{S}+\Gamma_{D}\right) d t .
$$

Note that an identical expression is obtained for $p_{S}(t)$. The time the particle spends in the dot, given that it eventually tunnels to the drain, is then

$$
\left\langle\tau_{+}^{(1)}\right\rangle \equiv \int_{0}^{\infty} d t t p(t \mid D)=\frac{1}{\Gamma_{D}+\Gamma_{S}} .
$$

We conclude that the time the electron spends in the dot is independent of the condition of eventually exiting to the left or to the right.

Assessing the sequential tunneling time [Eq. (A4)] by employing strong measurement protocol is straightforward. One can correlate the entry of an electron to the QD (detected through a clear signal in the QPC [23]) with the detection of the electron at the drain: the relevant quantity is $p(t \mid D)$. This becomes trickier when weak measurement by the detector is employed. One then needs to resort to Eq. (10), invoking the correlation function $S_{I J}$. We can determine the time resulting from Eq. (10) assuming, without loss of generality, that the current $J^{(1)}(s)=J+\xi(s)$ when the electron is in the dot, and $J^{(0)}(s)=0+\xi(s)$ otherwise (a constant reference current has been subtracted). $\xi(s)$ is a stochastic component of the current due to the detector's intrinsic noise with $\int_{-\infty}^{t} d s \xi(s)=0$, and it is uncorrelated to the QD signal. We also note that the event of having a current peak in the drain at time $t$ happens with probability $P(D \mid t)$ and the average in $S_{I J}$ has to be taken with respect to this probability. Measuring the current through the 
QD in units of the electron charge, we can write Eq. (10) as

$$
\begin{aligned}
S_{I J} & =P(t=0) \int_{0}^{\infty} d t \int_{0}^{t} d s[J P(D \mid t) p(t)+\xi(s)] \\
& =\left(\Gamma_{S} / f_{0}\right) J \int_{0}^{\infty} d t t \Gamma_{D} e^{\left(\Gamma_{D}+\Gamma_{S}\right) t},
\end{aligned}
$$

where $P(t=0)=\Gamma_{S} / f_{0}$ is the probability of entering the dot at time $t=0$. In the first equality of Eq. (A5), the term involving $\xi(s)$ is not weighted by any probability since it is not correlated to the dynamics of the dot. In the second equality in Eq. (A5), we take into account that the time integral of that same term involving the stochastic fluctuations vanishes. We may further write $I=\Gamma_{S} \Gamma_{D} /\left(\Gamma_{S}+\Gamma_{D}\right)$. With this expression, Eq. (9) leads to

$$
\left\langle\tau_{+}^{(1)}\right\rangle=1 /\left(\Gamma_{S}+\Gamma_{D}\right),
$$

in full agreement with the result of a strong measurement.

\section{APPENDIX B: CALCULATION OF THE QD CORRELATION FUNCTION}

In this section, we discuss the calculation of $\langle I\rangle$ and $\langle I(t)[N(t-s)-\langle N\rangle]\rangle$ in the electron cotunneling regime. We address first the calculation of the average current, and then its generalization to the charge-current correlation function.

\section{Average current}

The Hamiltonian is presented in Sec. II. The calculations are done perturbatively in the tunneling Hamiltonian $H_{T}$, hence, we work in the interaction picture with respect to $H_{0}+H_{\text {int }}+H_{\mathrm{QPC}}$, and denote by - the operators in the interaction picture. The leading-order term in the perturbative calculation of the current is obtained to third order in $H_{T}$. The cotunneling current [8] in Eq. (4) is in turn proportional to $\gamma^{4}$ :

$$
\begin{aligned}
\langle I\rangle= & e \operatorname{Re}\left\langle\int_{-\infty}^{t} d s_{1} \int_{-\infty}^{s_{1}} d s_{2} \int_{-\infty}^{s_{2}} d s_{3}\left[\bar{T}_{D}(t) \bar{H}_{T}\left(s_{1}\right) \bar{H}_{T}\left(s_{2}\right) \bar{H}_{T}\left(s_{3}\right)+\bar{H}_{T}\left(s_{3}\right) \bar{H}_{T}\left(s_{2}\right) \bar{H}_{T}\left(s_{1}\right) \bar{T}_{D}(t)\right]\right. \\
& \left.-\int_{-\infty}^{t} d s_{1} \int_{-\infty}^{t} d s_{2} \int_{-\infty}^{s_{2}} d s_{3}\left[\bar{H}_{T}\left(s_{1}\right) \bar{T}_{D}(t) \bar{H}_{T}\left(s_{2}\right) \bar{H}_{T}\left(s_{3}\right)+\bar{H}_{T}\left(s_{3}\right) \bar{H}_{T}\left(s_{2}\right) \bar{T}_{D}(t) \bar{H}_{T}\left(s_{1}\right)\right]\right\rangle,
\end{aligned}
$$

where the average is intended on an unperturbed state (possibly a mixed one), described by a density matrix $\rho(t=-\infty)$.

Each of the $H_{T}$ terms in Eq. (B1) consists in fact of a sum of terms involving products of $T_{S}, T_{D}$. The system's state is initially (at $t=-\infty$ ) an eigenstate of $N, N_{S}, N_{D}$. It remains an eigenstate of the same operators after the application of each $H_{T}$ (note that $\left.\left[N, T_{S}\right]=T_{S},\left[N, T_{S}^{\dagger}\right]=-T_{S}^{\dagger},\left[N, T_{D}\right]=T_{D},\left[N, T_{D}^{\dagger}\right]=-T_{D}^{\dagger}\right)$. In fact, $H_{T}$ changes the system's state to a configuration with \pm 1 extra charge on the QD. The time dependence in the operators in Eq. (B1) can then be made explicit in terms of the time-evolution operator and computed employing Eq. (8). To be specific, let us address one of the terms appearing in Eq. (B1), namely,

$$
\langle I\rangle_{1} \equiv e \operatorname{Re}\left\langle\int_{-\infty}^{t} d s_{1} \int_{-\infty}^{s_{1}} d s_{2} \int_{-\infty}^{s_{2}} d s_{3} \bar{T}_{D}(t) \bar{T}_{S}^{\dagger}\left(s_{1}\right) \bar{T}_{D}^{\dagger}\left(s_{2}\right) \bar{T}_{S}\left(s_{3}\right)\right\rangle .
$$

The explicit time dependence of operators results then in

$$
\langle I\rangle_{1}=e \operatorname{Re} \operatorname{Tr}\left\{\int_{-\infty}^{t} d s_{1} \int_{-\infty}^{s_{1}} d s_{2} \int_{-\infty}^{s_{2}} d s_{3} T_{D}(t) e^{-i\left(E_{+l}+e V\right)\left(t-s_{1}\right)} T_{S}^{\dagger}\left(s_{1}\right) e^{+i e V\left(s_{1}-s_{2}\right)} T_{D}^{\dagger}\left(s_{2}\right) e^{-i E_{+l}\left(s_{2}-s_{3}\right)} T_{S}\left(s_{3}\right) \rho(-\infty)\right\},
$$

where the operators evolve in time through

$$
\mathcal{U}\left(t, t^{\prime}\right)=\mathcal{T}-\exp \left\{-i H_{0}\left(t-t^{\prime}\right)\right\},
$$

where $\mathcal{T}$ is the time-ordering operator, and $H_{0}$ is the Hamiltonian of the dot-leads part, which includes neither the charging energy nor the detector Hamiltonian. The fact that the detector Hamiltonian is neglected in Eq. (B4) is a consequence of the perturbative calculation in the strength of the measurement. More precisely, since Eq. (13) is obtained by already computing $S_{I J}$ to first order in $H_{\text {int }}$, the $S_{I J}$ can be safely neglected in the calculation of $\langle I(t)[N(t-s)-\langle N\rangle]\rangle$. A proper treatment of $H_{\text {int }}$ in the calculation of $\langle I\rangle$ reveals that it makes contributions in second order, and is therefore consistently neglected here [30].

The quantum average in Eq. (B2) can now be easily obtained via Wick's theorem. This is conveniently done in terms of Feynman diagrams on the Keldysh contour, resulting in the rules specified in Appendix B 3. All possible diagrams correspond to all possible sequences of $T_{\alpha}\left(s_{j}\right)$ obtained from the $H_{T}$ in Eq. (B1), and are presented in Fig. 3.

Each diagram corresponds to a well-defined process which contributes to the probability of charge transfer between the two leads. The amplitude for a charge transfer is a superposition of electronlike $(e)$ and holelike $(h)$ processes. The various contributions to the probability are labeled accordingly (e.g., $e-e, e-h, \ldots$ ). Moreover, the diagrams are classified as forward (backward) when a charge is transferred from the source (drain) to the drain (source). For instance, the diagrams obtained by averaging the expression for $\langle I\rangle_{1}$ in Eq. (B2) are the " $e-h$ forward" both elastic and inelastic, according to the labeling in Fig. 3.

The analytical expression for each diagram is obtained by the rules R7-R10 in Appendix B 3. The backward diagrams are vanishing in the $T=0$ limit due to the vanishing of the corresponding phase space. Moreover, by controlling the gate voltage, one can tune the ratio $E_{-r} / E_{+l}$ in the regime 
(a)

Elastic
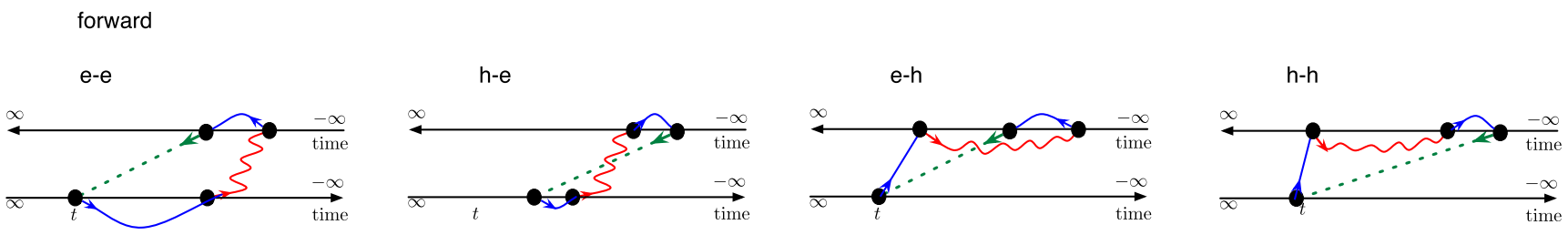

backward
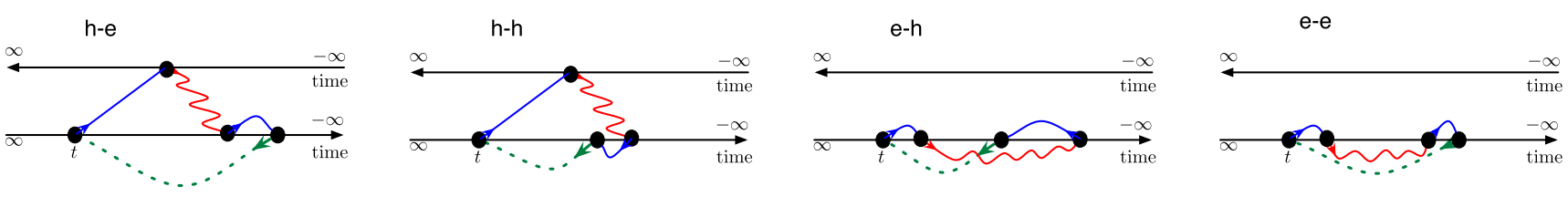

(b)

Inelastic


FIG. 3. (Color online) Feynman diagrams contributing to the cotunneling current. Each contribution to the probability consists of a coherent superposition of electronlike $(e)$ and holelike $(h)$ amplitudes. Upon squaring these amplitudes one obtains contributions of the type $e$ - $e$, $e$ - $h$, etc., which are represented by the various diagrams. The diagrams are grouped into elastic (A) and inelastic (B) contributions. Each group consists of "forward" and "backward" diagrams, depending on whether the associated charge transfer is from source to drain or vice versa, respectively. The contribution of the latter set of diagrams is vanishing at $T=0$. The relative weight of the electron and hole contributions is controlled by the gate voltage.

$E_{-r} / E_{+l} \ll 1$, where the holelike processes are parametrically suppressed by a factor $\mathcal{O}\left(E_{-r} / E_{+l}\right)$. In this zero-temperature particle-dominated limit, the elastic and inelastic contributions to the current are given by

$$
\begin{aligned}
& I_{\mathrm{el}}=\underbrace{\infty}_{\xi^{-\infty}}+\text { H.c. }=-2 e \operatorname{Re}\left\{\int_{\mathbb{R}_{+}^{3}} d x d y d z \sum_{\alpha, \beta, j, k}\left(1-n_{\alpha}\right)\left(1-n_{j}\right)\left(1-n_{\beta}\right) n_{k} \gamma_{\alpha, k}^{(S)} \gamma_{\alpha, j}^{(D)^{*}} \gamma_{\beta, j}^{(D)} \gamma_{\beta, k}^{(S)^{*}}\right. \\
& \left.\times e^{-i\left(E_{+, l}+\epsilon_{\alpha}-\epsilon_{k}\right) x} e^{-i\left(-e V+\epsilon_{j}-\epsilon_{k}\right) y} e^{i\left(E_{+, l}+\epsilon_{\beta}-\epsilon_{k}\right) z}\right\}, \\
& I_{\text {in }}=\underset{\infty}{\infty} \sum_{-\infty}^{-\infty}+\text { H.c. }=-2 e \operatorname{Re}\left\{\int_{\mathbb{R}_{+}^{3}} d x d y d z \sum_{\alpha, \beta, j, k}\left(1-n_{\alpha}\right)\left(1-n_{j}\right) n_{\beta} n_{k}\left|\gamma_{\alpha, k}^{(S)}\right|^{2}\left|\gamma_{\beta, j}^{(D)}\right|^{2}\right. \\
& \left.\times e^{-i\left(E_{+l}+\epsilon_{\alpha}-\epsilon_{k}\right) x} e^{-i\left(-e V+\epsilon_{\alpha}-\epsilon_{\beta}+\epsilon_{j}-\epsilon_{k}\right) y} e^{i\left(E_{+l}+\epsilon_{\alpha}-\epsilon_{k}\right) z}\right\},
\end{aligned}
$$

with $n_{\eta}$ the distribution function of the occupation of the $\eta$ th energy level. 
To proceed further, one notes that the tunneling matrix elements can be written in terms of the dot's and leads' wave functions $\psi(\mathbf{x})$ and $\phi(\mathbf{x})$, respectively, as $\gamma_{\alpha, k}^{(S)}=\sqrt{\mathcal{V} \mathcal{S}} \gamma \psi_{\alpha}\left(\mathbf{x}_{S}\right) \phi_{k}\left(\mathbf{x}_{S}\right)$, where $\mathbf{x}_{S}$ is the coordinate of the tunneling point between the source and the dot, $\mathcal{V}$ the volume of each lead, and $\mathcal{S}$ that of the dot. An equivalent expression holds for the drain. These are realizationdependent quantities, and we consider their statistical average. Independently of the QD dynamics, $\mathcal{V}\left\langle\phi_{k}^{(S) *}\left(\mathbf{x}_{S}\right) \phi_{k}^{(S)}\left(\mathbf{x}_{S}\right)\right\rangle=1$. Introducing the density of states in the leads $\sum_{k} \rightarrow \int_{\mathbb{R}} d \epsilon_{k} \nu$ and in the $\operatorname{dot} \sum_{\alpha} \rightarrow \int_{\mathbb{R}} d \epsilon_{\alpha} \nu_{0}$, the integrals in Eqs. (B5) and (B6) are finally performed to obtain

$$
\begin{gathered}
I_{\mathrm{in}}=e \frac{G^{(S)} G^{(D)}}{2 \pi e^{4}} \int_{\mathbb{R}+^{4}} d \epsilon_{\alpha} d \epsilon_{\beta} d \epsilon_{j} d \epsilon_{k} \frac{\delta\left(\epsilon_{\alpha}+\epsilon_{\beta}+\epsilon_{j}+\epsilon_{k}-e V\right) \mathcal{S}^{2}\left\langle\psi_{\alpha}\left(\mathbf{x}_{S}\right) \psi_{\alpha}^{*}\left(\mathbf{x}_{S}\right) \psi_{\beta}\left(\mathbf{x}_{D}\right) \psi_{\beta}^{*}\left(\mathbf{x}_{D}\right)\right\rangle}{\left(E_{C}+\epsilon_{\alpha}+\epsilon_{k}\right)^{2}}, \\
I_{\mathrm{el}}=e \frac{G^{(S)} G^{(D)}}{2 \pi e^{4}} e V \int_{0}^{\infty} d \epsilon_{\alpha} \int_{0}^{\infty} d \epsilon_{\beta} \frac{\mathcal{S}^{2}\left\langle\psi_{\alpha}\left(\mathbf{x}_{\mathbf{S}}\right) \psi_{\alpha}^{*}\left(\mathbf{x}_{D}\right) \psi_{\beta}\left(\mathbf{x}_{\mathbf{D}}\right) \psi_{\beta}^{*}\left(\mathbf{x}_{S}\right)\right\rangle}{\left(E_{C}+\epsilon_{\alpha}\right)\left(E_{C}+\epsilon_{\beta}\right)}+\mathcal{O}\left[(e V)^{2}\right],
\end{gathered}
$$

where $G^{(a)} \equiv e^{2} v v_{0}\left|\gamma^{(a)}\right|^{2} /(2 \pi \hbar)$ is the conductance of the $a=S, D$ contact.

The averages over the statistical realizations in Eqs. (B8) and (B7) are well known in the literature [29]. In the diffusive limit $L>\left|\mathbf{x}_{\mathbf{S}}-\mathbf{x}_{\mathbf{D}}\right| \gg l, \quad \mathcal{S}^{2}\left\langle\psi_{\alpha}{ }^{*}\left(\mathbf{x}_{S}\right) \psi_{\alpha}\left(\mathbf{x}_{S}\right) \psi_{\beta}{ }^{*}\left(\mathbf{x}_{D}\right)\right.$ $\left.\psi_{\beta}\left(\mathbf{x}_{D}\right)\right\rangle \approx 1$, and $\mathcal{S}^{2}\left\langle\psi_{\alpha}\left(\mathbf{x}_{S}\right) \psi_{\alpha}^{*}\left(\mathbf{x}_{D}\right) \psi_{\beta}\left(\mathbf{x}_{D}\right) \psi_{\beta}^{*}\left(\mathbf{x}_{S}\right)\right\rangle \equiv$ $\mathcal{D}_{\omega}\left(\mathbf{x}_{S}, \mathbf{x}_{D}\right) / \nu_{0}$, where $\mathcal{D}_{\omega}\left(\mathbf{x}_{S}, \mathbf{x}_{D}\right)$ is the diffuson propagator [29] between the source and the drain points, and $\omega=\epsilon_{\alpha}-\epsilon_{\beta}$. The cotunneling current then reads as $[8,9,25,29]$

$$
\begin{gathered}
I_{\text {in }}=\frac{G^{(S)} G^{(D)}}{12 \pi e^{2}} \frac{(e V)^{2}}{E_{C}^{2}} V \\
I_{\mathrm{el}}=\frac{G^{(S)} G^{(D)}}{4 \pi^{2} v_{0} e^{2}} V \int_{0}^{\infty} d \omega \frac{\mathcal{D}_{\omega}\left(\mathbf{x}_{S}, \mathbf{x}_{D}\right)+\mathcal{D}_{-\omega}\left(\mathbf{x}_{S}, \mathbf{x}_{D}\right)}{\omega} \\
\times \ln \left(1+\frac{\omega}{E_{C}}\right) .
\end{gathered}
$$

The elastic cotunneling current depends on the diffuson propagator, which is characterized by Thouless energy $E_{\mathrm{Th}} \sim D / \mathcal{S}$, proportional to the diffusion constant $D$. The cotunneling current depends on the ratio between $E_{\mathrm{Th}}$ and $E_{C}$. In the limit $E_{\mathrm{Th}} \gg E_{C}$ the elastic cotunneling current acquires the universal form

$$
I_{\mathrm{el}, 0 \mathrm{D}}=\frac{G^{(S)} G^{(D)}}{4 \pi e^{2}} \frac{\delta}{E_{C}} V .
$$

The expression is independent on the dot parameters and dynamics, and can be regarded as the expression for a "zerodimensional" (0D) dot. In the opposite limit, $E_{\mathrm{Th}} \ll E_{C}$, the result depends on the dot's shape and the electron's dynamics therein.

Addressing now the cotunneling time in the latter regime, we consider the cotunneling current in the specific case of a square dot of linear size $L$. We expect our result to be parametrically correct for other dot's shapes. The Thouless energy is then $E_{\mathrm{Th}}=D /\left(\pi^{2} L^{2}\right)$ and the diffuson is expressed by

$$
\mathcal{D}_{\omega}\left(\mathbf{x}_{S}, \mathbf{x}_{D}\right)=\sum_{\mathbf{n} \in \mathbb{N}^{+d}} \frac{S^{-1} \Phi\left(\mathbf{x}_{S}, \mathbf{x}_{D}\right)}{-i \omega+E_{\mathrm{Th}}|\mathbf{n}|^{2}},
$$

where $\mathbf{n}^{T}=\left(n_{1}, \ldots, n_{d}\right)$ in $d$ spatial dimensions, and $\Phi\left(\mathbf{x}_{S}, \mathbf{x}_{D}\right)=\left(1 / L^{d}\right) \prod_{j=1}^{d} \cos \left(\frac{n_{j} x_{S, j}}{\pi L}\right) \cos \left(\frac{n_{j} x_{D, j}}{\pi L}\right)$. We focus here on the case of a two-dimensional dot, although the calculation can be performed in general in any spatial dimensions [30]; we assume for simplicity $\mathbf{x}_{s}=\mathbf{0}$. The parameter $\eta \equiv \pi\left|\mathbf{x}_{S}-\mathbf{x}_{d}\right| \sqrt{E_{C} / D}$ discriminates between the two regimes $l / L \ll \eta \ll 1$ and $l / L \ll 1 \ll \eta$. In the latter case, the cotunneling current is known to be [8]

$$
I_{\mathrm{el}, \text { long }}=\frac{G^{(S)} G^{(D)}}{16 e^{2}} V \frac{\delta E_{\mathrm{Th}}}{E_{C}^{2}} .
$$

This limit corresponds to the case of the source and drain contact at the opposite sides of the dot. In the opposite regime of source and drain close to each other, we estimate the cotunneling current as

$$
I_{\mathrm{el}, \mathrm{short}} \approx \frac{G^{(S)} G^{(D)}}{8 \pi e^{2}} V \frac{\delta}{E_{\mathrm{Th}}} \int_{0}^{\infty} \frac{d x}{x} J_{0}(\eta x) f(x) .
$$

Here, $J_{0}(x)$ is the 0th Bessel function,

$$
\begin{aligned}
f(x)= & L i_{2}\left(\frac{1}{1-i x}\right)+L i_{2}\left(\frac{1}{1-i x}\right)+2 \pi \arctan (x) \\
& -\arctan ^{2}(x)-\frac{\pi^{2}}{3},
\end{aligned}
$$

and $-L i_{2}(-x)=\int_{0}^{x} d y \ln (1+y) / y$ is the dilogarithmic function. We are interested in the limit $\eta \ll 1$ where

$$
I_{\mathrm{el}, \text { short }} \approx \frac{G^{(S)} G^{(D)}}{12 \pi e^{2}} V \frac{\delta}{E_{\mathrm{Th}}} \ln ^{3}\left(\frac{D}{\pi^{2}\left|\mathbf{x}_{s}-\mathbf{x}_{D}\right|^{2} E_{C}}\right) .
$$

\section{Charge-current correlation function $\langle I(t) N(t-s)\rangle$}

The calculation presented above for the cotunneling current can be easily generalized to the correlation function $\int_{0}^{\infty} d s\langle I(t)[N(t-s)-\langle N\rangle]\rangle$. Given the specific time order between the operators, we focus on the calculation of $\mathcal{F}(s)=$ $\left\langle\mathcal{T}_{K}\left[I_{-}(t)\left[N_{+}(t-s)-\langle N\rangle\right]\right]\right\rangle$ written in terms of Keldysh time-ordered operators, and address the time integral later.

The calculation is done perturbatively in $\gamma$, in complete analogy with the case of the current. This leads to Feynman diagrams constructed according to the same rules R1-R6 discussed above. In fact, the diagrams obtained for the correlator can be easily deduced from the diagrams of the current. Each diagram contributing to the cotunneling current has its analog for the correlator at hand; the only difference between the two is the insertion of the vertex $N_{+}(t-s)$ in the upper branch. As presented in Fig. 4, this has two consequences: (i) the new $N$ vertex added to a certain current diagram can be connected in two different ways, leading correspondingly to two distinct diagrams for the correlation function; (ii) two diagrams of $I$ 

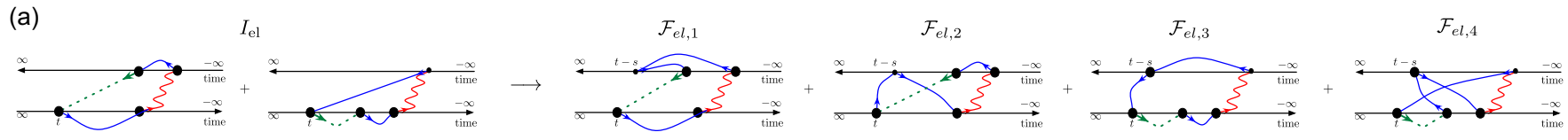

(b)
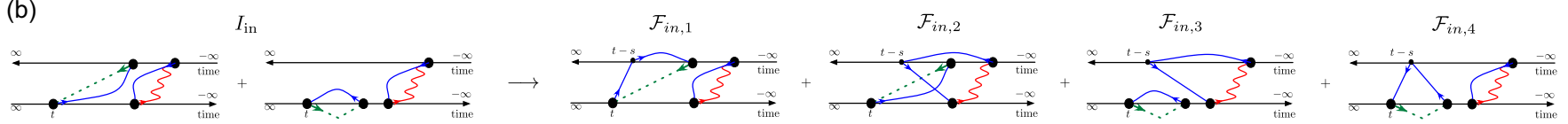

FIG. 4. (Color online) Example of Feynman diagrams for the correlation function $\left\langle I_{+}(t)\left[N_{-}(t-s)-\langle N\rangle\right]\right\rangle$ obtained from the corresponding diagrams for the cotunneling current. The presented diagrams are all those contributing the correlation function in the limit of zero-temperature and particle-dominated processes in the elastic (a) and inelastic (b) cotunneling regimes. For each diagram contributing the cotunneling current there are four diagrams contributing the correlation function.

related by complex conjugation become no longer the complex conjugate of each other when inserting the new $N$ vertex, so they have to be computed separately.

All the nonvanishing diagrams in the limit of zerotemperature and particle-dominated cotunneling are depicted in Fig. 4. Technically, the new propagator including an $N$ vertex, namely, $\left\langle\mathcal{T}_{K}\left\{c_{\alpha \pm}\left(s_{1}\right)\left[N_{+}(t-s)-\langle N\rangle\right] c_{\beta, \pm}^{\dagger}\left(s_{2}\right)\right\}\right\rangle$, can be directly evaluated. In the Schrödinger picture

$$
\left\langle c_{\alpha}(N-\langle N\rangle) c_{\beta}^{\dagger}\right\rangle=\delta_{\alpha, \beta}\left(1-n_{\alpha}\right)^{2}=\delta_{\alpha, \beta}\left(1-n_{\alpha}\right),
$$

$$
\begin{gathered}
\left\langle c_{\alpha}^{\dagger}(N-\langle N\rangle) c_{\beta}\right\rangle=-\delta_{\alpha, \beta} n_{\alpha}^{2}=-\delta_{\alpha, \beta} n_{\alpha}^{2}, \\
\left\langle c_{\alpha}^{\dagger}(N-\langle N\rangle) c_{\beta}^{\dagger}\right\rangle=\left\langle c_{\alpha}(N-\langle N\rangle) c_{\beta}\right\rangle=0,
\end{gathered}
$$

where the last equalities in Eqs. (B17) and (B18) are valid at $T=0$. In fact, the above equations can be effectively implemented in the Feynman diagrams as expressed in the rule R10. It immediately follows that, among all the contributing diagrams in Fig. $4, \mathcal{F}_{\mathrm{el}, 2}=\mathcal{F}_{\mathrm{el}, 4}=\mathcal{F}_{\mathrm{in}, 4}=0$. As an example, the nonvanishing diagram $\mathcal{F}_{\text {in, } 1}(s)$ in Fig. 4 reads as

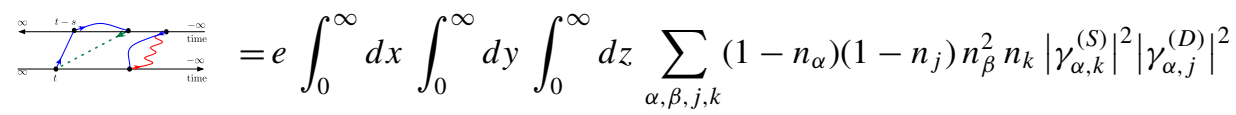

$$
\begin{aligned}
& \times e^{-i\left(E_{+, l}+\epsilon_{\alpha}+\epsilon_{k}\right) x} e^{-i\left(-e V+\epsilon_{\alpha}+\epsilon_{k}+\epsilon_{\beta}+\epsilon_{j}\right) y} e^{i\left(E_{+, l}+\epsilon_{\alpha}+\epsilon_{k}\right) z} e^{-i\left(-e V+\epsilon_{\alpha}+\epsilon_{k}+\epsilon_{\beta}+\epsilon_{j}\right) s} .
\end{aligned}
$$

A direct evaluation of all the diagrams in the inelastic and elastic regimes, done in complete analogy with the calculation of the current, leads to

$$
\begin{array}{r}
\mathcal{F}_{\text {in }}(s)=\mathcal{F}_{\text {in }, 1}(s)+\mathcal{F}_{\text {in }, 2}(s)+\mathcal{F}_{\text {in }, 3}(s)=-i e \frac{G^{(S)} G^{(D)}}{2 \pi e^{4}} \int_{\mathbb{R}_{+}^{4}} d \epsilon_{\alpha} d \epsilon_{\beta} d \epsilon_{j} d \epsilon_{k} L^{2}\left\langle\psi_{\alpha}\left(\mathbf{x}_{S}\right) \psi_{\alpha}^{*}\left(\mathbf{x}_{S}\right) \psi_{\beta}\left(\mathbf{x}_{D}\right) \psi_{\beta}^{*}\left(\mathbf{x}_{D}\right)\right\rangle \\
\times \frac{e^{-i\left(E_{C}+\epsilon_{\alpha}+\epsilon_{k}\right) s}}{\left(E_{C}+\epsilon_{\alpha}+\epsilon_{k}\right)^{2}}\left[\frac{1-e^{-i\left(\epsilon_{\beta}+\epsilon_{j}-E_{C}-e V\right) s}}{\epsilon_{\alpha}+\epsilon_{\beta}+\epsilon_{j}+\epsilon_{k}-e V+i \zeta}\right] \\
\mathcal{F}_{\mathrm{el}}(s)=\mathcal{F}_{\mathrm{el}, 1}(s)+\mathcal{F}_{\mathrm{el}, 3}(s)=i e \frac{G^{(S)} G^{(D)}}{2 \pi e^{4}} \int_{\mathbb{R}_{+}^{4}} d \epsilon_{\alpha} d \epsilon_{\beta} d \epsilon_{j} d \epsilon_{k} L^{2}\left\langle\psi_{\alpha}\left(\mathbf{x}_{S}\right) \psi_{\alpha}^{*}\left(\mathbf{x}_{D}\right) \psi_{\beta}\left(\mathbf{x}_{D}\right) \psi_{\beta}^{*}\left(\mathbf{x}_{S}\right)\right\rangle \\
\quad \times \frac{1}{\left(E_{C}+\epsilon_{\beta}+\epsilon_{k}\right)\left(E_{C}+\epsilon_{\alpha}+\epsilon_{k}\right)}\left[\frac{1-e^{-i\left(\epsilon_{\alpha}+\epsilon_{j}-E_{C}-e V\right) s}}{\epsilon_{j}+\epsilon_{k}-e V+i \zeta}+\frac{1}{\epsilon_{j}-\epsilon_{\alpha}-E_{C}-e V}\right]
\end{array}
$$

with an infinitesimal regularization parameter $\zeta$. After integrating over $s$ (eventually including a convergence factor $e^{-\zeta s}$ ), we obtain

$$
\begin{array}{r}
\int_{0}^{\infty} d s \mathcal{F}_{\mathrm{in}}(s)=i e \frac{G^{(S)} G^{(D)}}{2 \pi e^{4}} \int_{\mathbb{R}+^{4}} d \epsilon_{\alpha} d \epsilon_{\beta} d \epsilon_{j} d \epsilon_{k} \frac{\delta\left(\epsilon_{\alpha}+\epsilon_{\beta}+\epsilon_{j}+\epsilon_{k}-e V\right) L^{2}\left\langle\psi_{\alpha}\left(\mathbf{x}_{S}\right) \psi_{\alpha}^{*}\left(\mathbf{x}_{S}\right) \psi_{\beta}\left(\mathbf{x}_{D}\right) \psi_{\beta}^{*}\left(\mathbf{x}_{D}\right)\right\rangle}{\left(E_{C}+\epsilon_{\alpha}+\epsilon_{k}\right)^{3}} \\
\int_{0}^{\infty} d s \mathcal{F}_{\mathrm{el}}(s)=-i e \frac{G^{(S)} G^{(D)}}{4 \pi e^{4}} e V \int_{\mathbb{R}_{+}^{2}} d \epsilon_{\alpha} d \epsilon_{\beta} \frac{L^{2}\left\langle\psi_{\alpha}\left(\mathbf{x}_{S}\right) \psi_{\alpha}^{*}\left(\mathbf{x}_{D}\right) \psi_{\beta}\left(\mathbf{x}_{D}\right) \psi_{\beta}^{*}\left(\mathbf{x}_{S}\right)\right\rangle}{\left(E_{C}+\epsilon_{\beta}\right)\left(E_{C}+\epsilon_{\alpha}\right)^{2}}
\end{array}
$$

Indeed, Eqs. (B23) and (B24) show that $\int_{0}^{\infty} d s \mathcal{F}_{\text {in(el })}=$ $-(+) \partial_{E_{C}} I_{\text {in(el) }}$. This directly gives the relation between the cotunneling time and the cotunneling current in Eq. (14).

\section{Rules for Feynman diagrams}

As discussed in this appendix, the correlation function $\langle I(t) N(t-s)\rangle$ and the cotunneling current $\langle I\rangle$ are calculated 
(a)

\begin{tabular}{|c|}
\hline time \\
\hline
\end{tabular}

(b)

$N=\left\{T_{S}=\left\{T_{D}=\oint_{k} \quad \frac{t}{h} \frac{t^{\prime}}{h^{\prime}}=\delta_{h, h^{\prime}} e^{-i \epsilon_{0, h}\left(t^{\prime}-t\right)} n(h)\right.\right.$

FIG. 5. (Color online) (a) Time contour for the Keldysh formalism. (b) Rules for drawing vertices in the diagrams for $\langle I\rangle$ and $\langle I(t) N(t-s)\rangle$, required by the rule R2. (c) Explicit expressions of propagators stipulated by the rule $\mathrm{R} 8$.

perturbatively in $H_{T}$. They are obtained to fourth order in perturbation theory. The various contributions are expressed in terms of Feynman diagrams. We present here the rules to obtain all the diagrams for the correlation function and their corresponding analytical expression. The diagrams (Figs. 2, 3, and 4) are drawn on the Keldysh contour [cf. Fig. 5(a))]. The Feynman rules are as follows. R1: Each operator is drawn as a vertex on the Keldysh contour according to the expressions given in Fig. 5(b); the vertices for $T_{S}^{\dagger}, T_{D}^{\dagger}$ are obtained by reversing the arrows in $T_{S}$ and $T_{D}$. R2: Each operator (vertex) is labeled by a time and a subscript $(+$ or - ) indicating whether the operator appears in forward- or backward-in-time branch of the Keldysh contour. R3: To begin, the diagrams for the current $\langle I\rangle$ are drawn by inserting the operator $T_{D-}(t)$ or $T_{D-}^{\dagger}(t)$; the diagrams for the correlator $\langle I(t) N(t-s)\rangle$ require instead $T_{D-}(t)$ [or $T_{D_{-}^{\dagger}}^{\dagger}(t)$ ] and $N_{+}(t-s)$. R4: All possible combinations of $T_{S_{ \pm}}^{\dagger}\left(s_{2}\right), T_{D \pm}^{\dagger}\left(s_{3}\right)$ should be inserted, such that the inequality $s_{3}>s_{2}>s_{1}$ is satisfied by the operators appearing in the same branch. R5: Vertices should be connected in all possible ways through the appropriate propagators. R6: Each time interval between two subsequent vertices is labeled by the corresponding charging energy $\langle U\rangle$ (cf. Sec. II). This charging energy is set to 0 at $t=-\infty$. Following the time contour, each vertex changes the value of this energy in a well-defined definite way. We label the charging energy at a certain time in terms of the vertices that precede that time on the contour. We thus introduce $\left\langle U\left(T_{S}\right)\right\rangle=E_{C}$ and $\left\langle U\left(T_{D}^{\dagger}\right)\right\rangle=E_{C}^{\prime}$ (cf. Fig. 5 ). All the other charging energies are determined in terms of these two values. In particular, $\left\langle U\left(T_{D}^{\dagger} T_{S}\right)\right\rangle=-e V$ and $\left\langle U\left(T_{S}^{\dagger} T_{D}\right)\right\rangle=e V$. An example of energy labeling is shown in the diagram in the inset of Fig. 2.

So far, we have listed the rules for drawing and labeling the diagrams. We complement this list by the rules for calculating these diagrams. R7: Each vertex corresponds to $T_{D} \rightarrow \sum_{k h} T_{k, h}^{(r)}, T_{S} \rightarrow \sum_{k h} T_{k, h}^{(l)}$, and the respective complexconjugate expressions for $T_{S}^{\dagger}, T_{D}^{\dagger}$. R8: The propagators associated with the dot's dynamics are given by the expressions in Fig. 5(c); analogous expressions hold for the leads' propagators, where the energy $\epsilon_{0}$ is replaced by the energy of the modes in the lead $\epsilon_{k}^{(\alpha)}$. R9: A factor $e^{-i U\left(t-t^{\prime}\right)}$ should be included for the corresponding charging energy $\langle U\rangle$ between times $t$ and $t^{\prime}$. R10: Integration over times should be executed, accounting for the inequalities of $\mathrm{R} 4$.
[1] A. Messiah, Quantum Mechanics (Dover, New York, 1999).

[2] Y. Aharonov, D. Z. Albert, and L. Vaidman, Phys. Rev. Lett. 60, 1351 (1988).

[3] Y. Aharonov and D. Rohrlich, Quantum Paradoxes (Wiley, Hoboken, NJ, 2005).

[4] O. Hosten and P. Kwiat, Science 319, 787 (2008).

[5] P. B. Dixon, D. J. Starling, A. N. Jordan, and J. C. Howell, Phys. Rev. Lett. 102, 173601 (2009).

[6] J. S. Lundeen, B. Sutherland, A. Patel, C. Stewart, and C. Bamber, Nature (London) 474, 188 (2011).

[7] O. Zilberberg, A. Romito, D. J. Starling, G. A. Howland, C. J. Broadbent, J. C. Howell, and Y. Gefen, Phys. Rev. Lett. 110, 170405 (2013).

[8] D. Averin and Y. Nazarov, Phys. Rev. Lett. 65, 2446 (1990).

[9] L. Glazman and M. Pustilnik, in Nanophysics: Coherence and Transport, Lecture Notes of the Les Houches Summer School 2004, Session LXXXI, edited by H. Bouchiat, Y. Gefen, S. Gueron, G. Montambaux, and J. Dalibard (Elsevier, Amsterdam, 2005).

[10] I. L. Aleiner, P. W. Brouwer, and L. I. Glazman, Phys. Rep. 358, 309 (2002)

[11] L. Mandelstam and I. Tamm, J. Phys. (USSR) 9, 249 (1945).

[12] E. Condon and P. Morse, Rev. Mod. Phys. 3, 43 (1931).

[13] E. Wigner, Phys. Rev. 98, 145 (1955).

[14] M. Büttiker and R. Landauer, Phys. Rev. Lett. 49, 1739 (1982).
[15] M. Büttiker, Phys. Rev. B 27, 6178 (1983).

[16] K. Mullen, E. Ben-Jacob, Y. Gefen, and Z. Schuss, Phys. Rev. Lett. 62, 2543 (1989).

[17] D. Sokolovski and L. M. Baskin, Phys. Rev. A 36, 4604 (1987).

[18] D. Sokolovski and J. Connor, Phys. Rev. A 47, 4677 (1993).

[19] A. M. Steinberg, Phys. Rev. Lett. 74, 2405 (1995).

[20] Y. Choi and A. N. Jordan, Phys. Rev. A 88, 052128 (2013).

[21] M. Field, C. G. Smith, M. Pepper, D. A. Ritchie, J. E. F. Frost, G. A. C. Jones, and D. G. Hasko, Phys. Rev. Lett. 70, 1311 (1993).

[22] L. DiCarlo, H. J. Lynch, A. C. Johnson, L. I. Childress, K. Crockett, C. M. Marcus, M. P. Hanson, and A. C. Gossard, Phys. Rev. Lett. 92, 226801 (2004).

[23] E. V. Sukhorukov, A. N. Jordan, S. Gustavsson, R. Leturcq, T. Ihn, and K. Ensslin, Nat. Phys. 3, 243 (2007).

[24] A. N. Korotkov and D. V. Averin, Phys. Rev. B 64, 165310 (2001).

[25] I. L. Aleiner, N. S. Wingreen, and Y. Meir, Phys. Rev. Lett. 79 , 3740 (1997).

[26] Y. Blanter and M. Büttiker, Phys. Rep. 336, 1 (2000).

[27] A. Kamenev, in Nanophysics: Coherence and Transport, Lecture Notes of the Les Houches Summer School 2004, Session LXXXI, edited by H. Bouchiat, Y. Gefen, S. Gueron, G. Montambaux, and J. Dalibard (Elsevier, Amsterdam, 2005).

[28] L. V. Keldysh, Zh. Eksp. Teor. Fiz. 47, 1515 (1965) [Sov. Phys. JETP 20, 1018 (1965)].

[29] A. Mirlin, Phys. Rep. 326, 259 (2000).

[30] A. Romito and Y. Gefen (unpublished). 